\title{
Laparoscopic Ovarian Cystectomy in A Patient With Vetriculoperitoneal Shunt: Case Report
}

\author{
Shrestha $\mathbf{R}^{1}$, Bhandari $\mathbf{B R}^{\mathbf{1}}$, Piya $\mathbf{R}^{\mathbf{2}}$, Shrestha $\mathbf{M R}^{3}$ \\ ${ }^{1} \mathrm{MD}$, Department of Obstetrics and Gynaecology, ${ }^{2} \mathrm{MD}$, Department of Anaesthesiology, ${ }^{3} \mathrm{MS}$, Department of Surgery, \\ Midat Hospital, Lagankhel, Lalitpur, Nepal
}

Received: 12-Nov-2016; Accepted: 17-Dec-2016

Ventriculoperitoneal shunt (VPS) is often the best treatment modality for neurologic disorders such as hydrocephalus and pseudotumor cerebri. In recent years, laparoscopic surgery has emerged as a preferred method of accessing and treating a variety of abdominopelvic pathology. Laparoscopic procedures were previously contraindicated in patients of hydrocephalus having a Ventriculoperitoneal (VP) shunt due to shunt obstruction, shunt malfunction, infection and acute elevation of intracranial pressure. Now, conclusive evidence indicating that it is safe to perform laparoscopic surgery in a patient with VP shunt using routine anaesthetic monitoring and without any shunt manipulation. We report a case of successfully performing laparoscopic ovarian cystectomy in a patient with VP shunt.

Keywords: intracranial pressure; laparoscopy; pneumoperitoneum; ventriculoperitoneal shunt.

\section{INTRODUCTION}

Hydrocephalus is an abnormal build-up of the cerebrospinal fluid (CBF) in the ventricles of the brain. The incidence of hydrocephalus is around 0.91.5 cases per 1000 births. The aetiology is variable and includes acqueductal stenosis, myelomeningocele, intraventricular bleeding, meningitis, tumour, and head injury. The treatment of hydrocephalus involves the shunting of cerebrospinal fluid to another area of the body, which is commonly the abdominal cavity. Ventriculoperitoneal shunting is the most common approach because the placement of the distal catheter is simple from a technical standpoint and surgical revision is rare. ${ }^{1}$ The other routes that are used are ventriculo-atrial, ventriculo-pleural, and lumbarperitoneal. Advances in surgeries of cerebral shunts and the increased life span associated with them, has made the incidence of patients with VP shunts presenting with an indication for laparoscopy higher. ${ }^{2}$ Laparoscopy has been a well-known procedure in the field of gynaecology and for many years, it has been used for female sterilization or as a diagnostic procedure for various acute and chronic abdominal ailments. In comparison to the standard laparotomy, small incision laparoscopic surgery offers unique advantages with respect to speedy recovery and

\section{CORRESPONDENCE}

Ranjana Shrestha

Consultant, Obstetrics/Gynaecology

MIDAT Hospital, Lagankhel, Lalitpur

Email: ranjana_514@hotmail.com

Phone: +977-9841817268 mobilization, reduced morbidity due to minimal surgical trauma and shorter hospital stay with its economic benefits. In view of its definite advantages to the patient as well as the hospital, laparoscopic surgery has progressed very rapidly and has become frequently performed procedure in gynaecology practice. $^{3}$

A Ventriculo-peritoneal shunt comprises of a silicone catheter stationed in the lateral cerebral ventricle, a reservoir, a unidirectional valve and a tube ending with a catheter lying freely in the peritoneal cavity. From the lateral ventricle the catheter is tunnelled through the subcutaneous tissue into the free peritoneal space in order to drain off the surplus cerebrospinal fluid. A patent and functional shunt relieves the raised ICP resulting from hydrocephalus by virtue of continuous drainage of the cerebrospinal fluid into the abdomen. The unidirectional valve is instrumental in preventing the reflux of cerebrospinal and intra-abdominal fluids. ${ }^{4}$ The shunt valve is capable of withstanding a pressure of up to $300 \mathrm{mg} \mathrm{Hg}$. Hence an intra abdominal pressure of $12-15 \mathrm{mmHg}$ which is used to insufflate the abdomen during laparoscopic ovarian cystectomy is very unlikely to produce pneumocephalus. ${ }^{5}$

\section{CASE}

A newly married, 25 years old lady, presented to the gynaecology department of MIDAT hospital, Lalitpur with complaints of pain lower abdomen for three months and pain over VP shunt placement site since four days. She gave a history of ventriculoperitoneal shunt placement four years back for the management of Idiopathic Intracranial Hypertension. 
Her general and systemic examinations were normal. On ultrasonography, there was a huge complex right adnexal cyst occupying whole pelvis, measuring $12 \times 9.5 \times 11 \mathrm{~cm}$ with no internal septations and no solid components. Her CA-125 was slightly raised (45units/ml). Her other relevant investigations were normal.

The patency of the shunt and its proper functioning of were preoperatively verified by the Neurosurgeon. Written Informed Consent was obtained from the patient for the laparoscopic procedure. The patient was planned for elective laparoscopic ovarian cystectomy after a thorough assessment by the anaesthesiologist. Laparoscopic ovarian cystectomy was carried out using a standard 4-port technique. The first port access was obtained in the lower margin of umbilicus by using Hasson's technique and the other ports were placed by inserting trocars under vision away from the shunt. The abdomen was insufflated with $\mathrm{CO} 2$ to a pressure of $12-15 \mathrm{mmHg}$.

On diagnostic laparoscopy, there was twice coiled VP shunt lying freely in the abdomen and pelvis without any adhesions. There was around $15 \mathrm{cmX} 10 \mathrm{~cm}$ thick walled right ovarian cyst occupying whole pelvis with almost $750 \mathrm{ml}$ mucinous fluid with no solid components. Uterus and bilateral fallopian tubes were normal with left ovary polycystic (Fig 1,2,3,4). Routine anesthetic monitoring took place all through the operation with special precautions. The surgery was completed laparoscopically within 60 minutes. The shunt was seen lying free and intact at the end of the procedure. Complete haemostasis was achieved. The patient tolerated the pnuemoperitoneum and the entire procedure with no hemodynamic instability to suggest increased intracranial pressure.

The patient's post-operative recovery was uneventful and was discharged on the second post operative day. Her histopathological examination report showed ovarian stroma tissue lined by cuboidal to low columnar mucinous epithelium with no features of atypia suggestive of mucinous cystadenoma of right ovary (Fig 5). There was no neurological deficit or signs of increased ICP in successive follow up till 2 month of surgery.

\section{COMMENT}

The main site of CSF formation is the choroid plexus, which produces 21 to $22 \mathrm{~mL}$ per hour or approximately $500 \mathrm{~mL}$ per day. The CSF is renewed every 4 to 5 days and serves primarily a mechanical buoyancy function. Because the brain and spinal cord have no lymphatic channels, a constant volumepressure relationship of the central nervous system is maintained by the CSF. Perturbations in this system may result in a rise in intracranial pressure (ICP) and a decrease in cerebral perfusion pressure (CPP) if mean arterial pressure (MAP) is held constant. If autoregulatory mechanisms are overcome or poorly functioning, this may result in decreased cerebral blood flow (CBF), with neurologic sequelae.?

The potential mechanism of increase in the intracranial pressure during laparoscopy is due to an increased outflow resistance or (though less likely) a retrograde flow. Theoretically, high intra-abdominal pressure may directly obstruct the antegrade cerebrospinal fluid flow and the retrograde passage of carbon dioxide gas may result in a sudden increase in intracranial pressure. The combined effect of hypercapniainduced cerebral arterial dilatation and venous pressure elevation generated by abdominal pressure may cause increased intracranial blood volume and increased intra-pressure in the fixed volume of the cranium. While the very original shunting equipment was quite similar to a simple catheter, quite soon a no-reflow valve was added. Thus the risk of a sharp rise of the intracranial pressure has possibly been over-evaluated. ${ }^{1}$

Figure 1: Laproscopic view showing VP shunt and right ovarian cyst

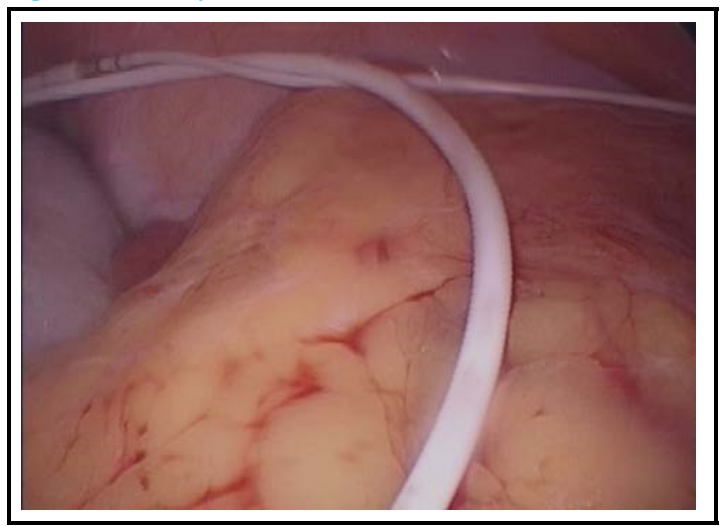

We have described a case of successful laparoscopic ovarian cystectomy in a patient of VP shunt without any evidence of raised intracranial pressure during and after surgery. Without laparotomy incisions, laparoscopy has been shown to decrease hospital stays, wound infection rates and narcotic usage as well as improve cosmesis. However, the application 
of laparoscopy in patients with VP has been questioned. ${ }^{6}$ The proposed complications related to using laparoscopic surgery in the presence of a VP or LP shunt include raised intracranial pressure (ICP), shunt malfunction and shunt infection. ${ }^{7}$ Jackman et al reported a retrospective review of urological laparoscopic surgery with standard anaesthetic monitoring in 18 patients with VP shunts which showed no untoward surgical, anaesthetic and neurological events. ${ }^{8}$

Figure 2: Showing aspiration of right ovarian cyst

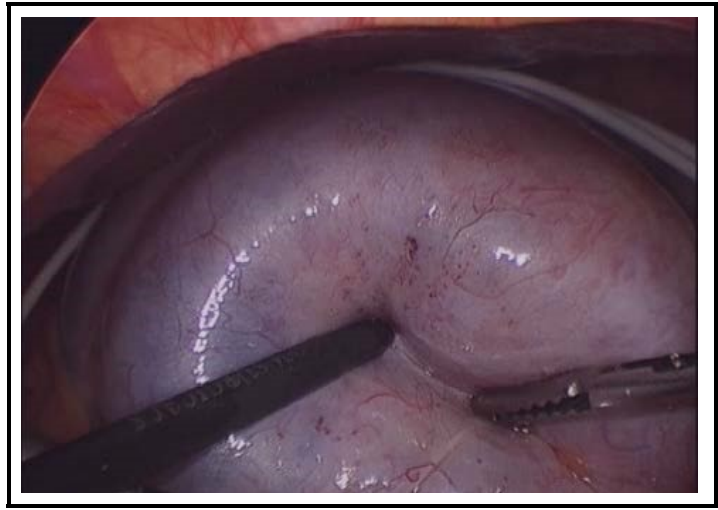

Figure 3: After enucleation of right ovarian cyst

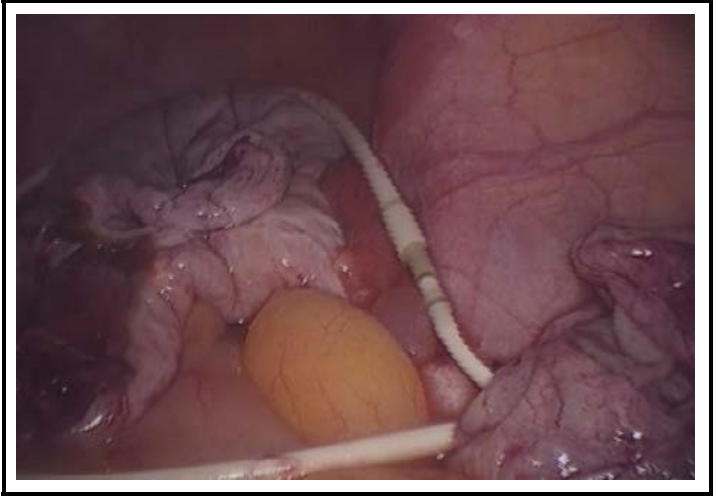

Figure 4: Excised right ovarian cyst and around $750 \mathrm{ml}$ mucinous fluid as the content of cyst

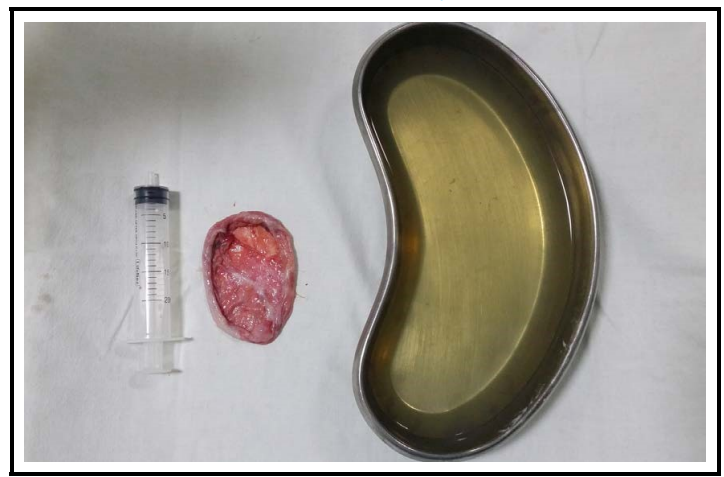

Baskin et al described a case of laparoscopic cholecystectomy in VP shunt patient who presented postoperatively with distal obstruction of the shunt thought to be due to soft tissue impaction or an air lock as a consequence of insufflation, which was easily cleared by tubing irrigation. ${ }^{9}$

Figure 5: Histopathological picture suggestive of mucinous cyst adenoma of right ovary

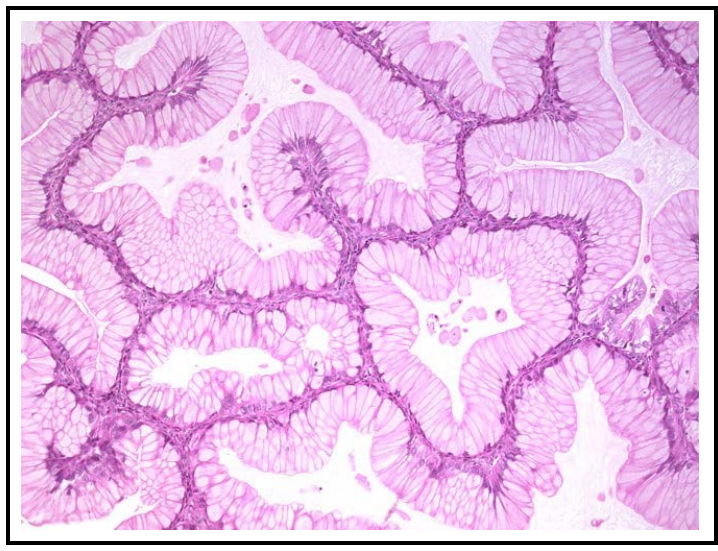

In a case of Schwed et al, respiratory compromise secondary to subcutaneous emphysema developed during a laparoscopic procedure performed just 10days after ventriculoperitoneal shunt placement. ${ }^{10}$ Ein et al performed a 30-year retrospective review at the Hospital for Sick Children in Toronto reviewing eight children with VPSs who underwent appendectomy. Three of the eight patients had a ruptured appendix. The surgeons externalized the shunts in each of these cases with future revision. The five patients with an unruptured appendix who did not have externalization of their shunt experienced no shunt complication. ${ }^{11}$

\section{CONCLUSIONS}

Based on the available data and this case we suggest that laparoscopic ovarian cystectomy can be safely performed on patients with a ventriculoperitoneal shunt. We also believe that a consultation with a neurosurgeon before the operation is advisable in order to verify the correct function of the shunt valve. 


\section{REFERENCES}

1. Cobianchi L, Dominioni T, Filisetti C, Zonta S, Maestri M, Dionigi P, Alessiani M. Ventriculoperitoneal shunt and the need to remove a gallbladder: Time to definitely overcome the feeling that laparoscopic surgery is contraindicated. Annals of Medicine and Surgery. 2014;3:65-7.

2. Sankpal R, Chandavarkar A, Chandavarkar M. Safety of Laparoscopy in Ventriculoperitoneal Shunt Patients. Journal of Gynecological Endoscopy and Surgery. 2011;2(2):91-3.

3. Dhaliwal JK, Shafei AA, Sharqi MA. Laparoscopic Surgery In Gynaecology - Salmaniya Experience. Bahrain Medical Bulletin. 2000;22(4):1-8

4. Chaudhari N, Khopde S, Thombare B, Shah HK Safety of laparoscopic cholecystectomy in patients with ventriculoperitoneal shunt. National Journal of Medical and Allied Sciences. 2014;3(1):36-9.

5. Collure DW, Bumpers HL, Luchette FA, Weaver WL, Hoover EL. Laparoscopic cholecystectomy in patients with ventriculoperitoneal (VP) shunts. Surg Endosc. 1995;9(4):409-10.
6. Fraser JD, Aguayo P, Sharp SW, Holcomb GW, Ostlie DJ, Peter SDS. The Safety of Laparoscopy in Pediatric Patients with Ventriculoperitoneal Shunts. Journal of Laparoendoscopic \& Advanced Surgical Techniques. 2009;19(5):675-8.

7. Uzzo RG, Bilsky M, Mininberg DT, Poppas DP. Laparoscopic surgery in children with ventriculoperitoneal shunts: effect of pneumoperitoneum on intracranial pressure-preliminary experience. Urology.1997;49(5):753-7.

8. Jackman SV, Weingart JD, Kinsman SL, Docimo SG. Laparoscopic surgery in patients with ventriculoperitoneal shunts: safety and monitoring. Journal of Urology. 2000;164(4):1352-4.

9. Baskin JJ, Vishteh AG, Wesche DE, Rekate HL, Carrion CA. Ventriculoperitoneal shunt failure as a complication of laparoscopic surgery. JSLS. 1998;2(2):177-80.

10. Schwed DA, Edoga JK, McDonnell TE. Ventilatory impairment during laparoscopic cholecystectomy in a patient with a ventriculoperitoneal shunt. J Laparoendosc Surg. 1992;2:57.

11. Ein S, Miller S, Rutka J. Appendicitis in the child with a ventriculoperitoneal shunt: a 30-year review. J Pediatr Surg. 2006;41:1255-8. 